\title{
Sobolev Inequalities, Riesz Transforms, and the Ricci Flow
}

\section{Rugang Ye}

Received: 31 July 2014 / Revised: 9 September 2014 / Accepted: 12 September 2014 /

Published online: 14 November 2014

(C) School of Mathematical Sciences, University of Science and Technology of China and Springer-Verlag Berlin Heidelberg 2014

\begin{abstract}
A number of results about deriving further Sobolev inequalities from a given Sobolev inequality are presented. Various techniques are employed, including Bessel potentials and Riesz transforms. Combining these results with the $W^{1,2}$ Sobolev inequality along the Ricci flow established by the author in earlier papers then yields various new Sobolev inequalities along the Ricci flow.
\end{abstract}

Keywords Ricci flow $\cdot$ Sobolev inequalities $\cdot$ Riesz transforms

Mathematics Subject Classification $53 \mathrm{C} 44 \cdot 46 \mathrm{E} 35$

\section{The Main Results}

In this section, we present the main results of this paper. Most proofs will be given in the subsequent sections. We divide the various results into several parts.

\subsection{Part A}

In [9-12], logarithmic Sobolev inequalities along the Ricci flow were obtained. As a consequence, $W^{1, p}$ Sobolev inequalities with $p=2$ along the Ricci flow were derived. Let $M$ be a closed manifold of dimension $n \geq 2$. Let $g=g(t)$ be a smooth solution of the Ricci flow

$$
\frac{\partial g}{\partial t}=-2 \text { Ric }
$$

\footnotetext{
R. Ye $(\bowtie)$

Department of Mathematics, University of California, Santa Barbara, CA, USA

e-mail: yer@math.ucsb.edu
} 
on $M \times[0, T)$ for some (finite or infinite) $T>0$. In the case $\lambda_{0}\left(g_{0}\right)>0$, where $\lambda_{0}\left(g_{0}\right)$ denotes the first eigenvalue of the operator $-\Delta+\frac{R}{4}$ of the initial metric $g_{0}=g(0)$, the Sobolev inequality takes the following form (in the case $n \geq 3$ )

$$
\left(\int_{M}|u|^{\frac{2 n}{n-2}} \mathrm{dvol}\right)^{\frac{n-2}{n}} \leq A \int_{M}\left(|\nabla u|^{2}+\frac{R}{4} u^{2}\right) \mathrm{dvol},
$$

where the positive constant $A$ depends on the initial metric in terms of rudimentary geometric data. If the condition $\lambda_{0}\left(g_{0}\right)>0$ is not assumed, then the Sobolev inequality takes the form (again in the case $n \geq 3$ )

$$
\left(\int_{M}|u|^{\frac{2 n}{n-2}} \mathrm{dvol}\right)^{\frac{n-2}{n}} \leq A \int_{M}\left(|\nabla u|^{2}+\frac{R}{4} u^{2}\right) \mathrm{dvol}+B \int_{M} u^{2} \mathrm{dvol},
$$

where the positive constants $A$ and $B$ depend on a finite upper bound for $T$ and the initial metric in terms of rudimentary geometric data. (If $\lambda_{0}\left(g_{0}\right)=0$, then the dependence on $T$ is not needed.)

As is well known, the case $p=2$ of the $W^{1, p}(M)$ Sobolev inequalities is used most often in analysis and geometry. However, it is of high interest to understand the situations $1<p<2$ and $2<p<n$, both from the point of view of a deeper understanding of the theory and the point of view of further applications. In this paper, we derive $W^{1, p}$ and $W^{2, p}$ Sobolev inequalities for general $p$ along the Ricci flow in several different ways. We will take a general point of view, and study the general problem of deriving further Sobolev inequalities from a given Sobolev inequality. In particular, we will include non-compact manifolds and manifolds with boundary, which require additional care. Our first result is the following one.

Theorem 1.1 Let $(M, g)$ be a Riemannian manifold of dimension $n \geq 2$, with or without boundary. (It is not assumed to be compact or complete.) Assume that for some $1 \leq p_{0}<n$ and non-negative constants $A$ and $B$ the inequality

$$
\left(\int_{M}|u|^{\frac{p_{0} n}{n-p_{0}}} \mathrm{dvol}\right)^{\frac{n}{n-p_{0}}} \leq A \int_{M}|\nabla u|^{p_{0}} \mathrm{dvol}+\frac{B}{\operatorname{vol}_{g}(M)^{\frac{p_{0}}{n}}} \int_{M}|u|^{p_{0}} \mathrm{dvol}
$$

holds true for all $u \in W^{1, p_{0}}(M)$. Then we have for all $p_{0}<p<n$ and $u \in W^{1, p}(M)$

$$
\left(\int_{M}|u|^{\frac{n p}{n-p}} \mathrm{dvol}\right)^{\frac{n-p}{n}} \leq C_{1} \int_{M}|\nabla u|^{p} \mathrm{dvol}+\frac{C_{2}}{\operatorname{vol}_{g}(M)^{\frac{p}{n}}} \int_{M}|u|^{p} \mathrm{dvol}
$$

where the positive constants $C_{1}=C_{1}\left(n, p_{0}, p, A, B\right)$ and $C_{2}=C_{2}\left(n, p_{0}, p, A, B\right)$ depend only on $n, p_{0}, p, A$ and $B$. Their dependence on $p$ is in terms of an upper bound for $\frac{1}{n-p}$. (If $\operatorname{vol}_{g}(M)=\infty$, then it is understood that the second term on the right hand side in (1.4) and (1.5) is zero, and $B$ is absent elsewhere.) 
This theorem is proved by an induction scheme based on the Hölder inequality. The principle that Sobolev inequalities of lower $p$ lead to Sobolev inequalities of higher $p$ is known. For example, it is well-known that the Sobolev inequality

$$
\|u\|_{\frac{n p}{n-p}} \leq C\|\nabla u\|_{p}
$$

for $u \in W_{0}^{1, p}\left(\mathbf{R}^{n}\right), 1<p<n$, can be derived from the case $p=1$, see e.g., [4]. However, the result in Theorem 1.1 is new, and the proof is more involved. Combining this theorem with the results in $[9,10]$ and [11] we then obtain $W^{1, p}$ Sobolev inequalities along the Ricci flow for $p>2$ in various situations. To keep this paper streamlined, we only state the results in the situation of [9]. The results in the situations of [10] and [11] are similar and obvious. In the following two theorems, we consider as before a smooth solution $g=g(t)$ of the Ricci flow on a closed manifold $M$ of dimension $n \geq 3$ for $0 \leq t<T$, where $T>0$ is allowed to be $\infty$.

Theorem 1.2 Assume $T<\infty$. Let $2<p<n$. Then there holds for each $t \in[0, T)$ and all $u \in W^{1, p}(M)$

$$
\left(\int_{M}|u|^{\frac{n p}{n-p}} \mathrm{dvol}\right)^{\frac{n-p}{n}} \leq A\left[1+\left(\max R^{+}+1\right) \operatorname{vol}(M)^{\frac{2}{n}}\right]^{\frac{r(p) p}{2}} \int_{M}\left(|\nabla u|^{p}+|u|^{p}\right) \mathrm{d} \operatorname{vol},
$$

where all geometric quantities are associated with $g(t)$, except the constant $A$, which can be bounded from above in terms of the dimension $n$, a non-positive lower bound for $R_{g_{0}}$, a positive lower bound for $\operatorname{vol}_{g_{0}}(M)$, an upper bound for $C_{S}\left(M, g_{0}\right)$, an upper bound for $T$, and an upper bound for $\frac{1}{n-p}$. The number $r(p)$ is defined in Theorem 2.1 below with $p_{0}=2$.

Theorem 1.3 Assume that $\lambda_{0}\left(g_{0}\right)>0$. Let $2<p<n$. Then there holds for each $t \in[0, T)$ and all $u \in W^{1, p}(M)$

$$
\left(\int_{M}|u|^{\frac{n p}{n-p}} \mathrm{dvol}\right)^{\frac{n-p}{n}} \leq A\left[\left(\max R^{+}+1\right) \operatorname{vol}(M)^{\frac{2}{n}}\right]^{\frac{r(p) p}{2}} \int_{M}\left(|\nabla u|^{p}+|u|^{p}\right) \mathrm{d} \operatorname{vol},
$$

where all geometric quantities are associated with $g(t)$, except the constant $A$, which can be bounded from above in terms of the dimension $n$, a non-positive lower bound for $R_{g_{0}}$, a positive lower bound for $\operatorname{vol}_{g_{0}}(M)$, an upper bound for $C_{S}\left(M, g_{0}\right)$, a positive lower bound for $\lambda_{0}\left(g_{0}\right)$, and an upper bound for $\frac{1}{n-p}$. The number $r(p)$ is the same as in Theorem 1.2.

A positive lower bound of $\lambda_{0}\left(g_{0}\right)$ implies an upper bound for $T$, hence Theorem 1.3 can be viewed as a corollary of Theorem 1.2. We state it as a separate theorem because of the geometric significance of this special case. Note that the distinction between (1.2) and (1.3) regarding the form of the Sobolev inequalities is not present between 
Theorem 1.2 and Theorem 1.3. The sole difference lies in the dependence of the constant $A$.

We would like to mention that these results as well as the other results in the subsequent parts regarding Sobolev inequalities along the Ricci flow easily extend (with some minor modifications) to the volume-normalized Ricci flow and the KählerRicci flow. (This is a straightforward consequence of the relevant results in [9].) They also extend to the Ricci flow with surgeries in dimension 3 as constructed in [6]. This will be presented elsewhere.

\subsection{Part B}

First we present a result on non-local Sobolev inequalities which is analogous to [9, Theorem C.5], but is formulated in terms of the canonical $(1, p)$-Bessel norm for Sobolev functions.

Definition Let $(M, g)$ be a metrically complete Riemannian manifold, with or without boundary. Let $1<p<\infty$. Let the Bessel-Sobolev space $L_{B}^{1, p}(M)$ be the completion of $C_{c}^{\infty}(M)$ with respect to the norm $\left\|(-\Delta+1)^{\frac{1}{2}}\right\|_{p}$. (See Sect. 3 for the construction of the operator $(-\Delta+1)^{\frac{1}{2}}$. ) We shall say that $g$ is a $p$-Bessel metric, and $(M, g)$ is a $p$-Bessel (Riemannian) manifold, if $L_{B}^{1, p}(M)$ is equivalent to $W^{1, p}(M)$, i.e.,

$$
c_{1}\|u\|_{1, p} \leq\left\|(-\Delta+1)^{\frac{1}{2}} u\right\|_{p} \leq c_{2}\|u\|_{1, p}
$$

for all $u \in C_{c}^{\infty}(M)$ and some positive constants $c_{1}$ and $c_{2}$, where $\|u\|_{1, p}=\|u\|_{p}+$ $\|\nabla u\|_{p}$ is the $W^{1, p}$ norm of $u$.

Assume that $(M, g)$ is $p$-Bessel. We define the $(1, p)$-Bessel norm for $f \in$ $W^{1, p}(M)$ to be $\|f\|_{B, 1, p}=\left\|(-\Delta+1)^{\frac{1}{2}} f\right\|_{p}$.

The operators $(-\Delta+1)^{\alpha}$ are called Bessel potentials, which is the reason for the above terminologies involving "Bessel". Note that every metrically complete $(M, g)$ is 2-Bessel because of the identity

$$
\left\langle(-\Delta+1)^{\frac{1}{2}} u,(-\Delta+1)^{\frac{1}{2}} u\right\rangle_{2}=\int_{M}\left(|\nabla u|^{2}+u^{2}\right) \mathrm{dvol}
$$

for $u \in C_{c}^{\infty}(M)$ (and then also for $u \in W^{1,2}(M)$ ), where $\langle\cdot, \cdot\rangle_{2}$ is the $L^{2}$ inner product. By the arguments in [9, Appendix C] (see also [8]), $(M, g)$ is $p$-Bessel for each $1<p<\infty$ if $M$ is compact.

Theorem 1.4 Let $(M, g)$ be a metrically complete manifold with or without boundary of dimension $n \geq 2$. Let $1<\mu<\infty$. Assume the Sobolev inequality

$$
\left(\int_{M}|u|^{\frac{2 \mu}{\mu-2}} \mathrm{dvol}\right)^{\frac{\mu-2}{\mu}} \leq A \int_{M}|\nabla u|^{2} \mathrm{dvol}+B \int_{M}|u|^{2} \mathrm{dvol}
$$


for all $u \in W^{1,2}(M)$. Let $1<p<\mu$. Then there holds

$$
\left\|(-\Delta+1)^{-\frac{1}{2}} u\right\|_{\frac{\mu p}{\mu-p}} \leq C(\mu, A, B, p)\|u\|_{p}
$$

for all $u \in L^{p}(M)$, where the constant $C(\mu, A, B, p)$ can be bounded from above in terms of upper bounds for $A, B, \mu, \frac{1}{\mu-p}$ and $\frac{1}{p-1}$. Consequently, there holds for a given $1<p<\mu$

$$
\|u\|_{\frac{\mu p}{\mu-p}} \leq C(\mu, A, B, p)\|u\|_{B, 1, p}
$$

for all $u \in W^{1, p}(M)$, provided that $(M, g)$ is $p$-Bessel.

Combining this theorem with the results in $[9,10]$ and [11] we obtain non-local Sobolev inequalities along the Ricci flow which are analogous to Theorem C.6 and C.7 in [9], but are formulated in terms of the canonical $(1, p)$-Bessel norm. Again we only state the results in the situation of [9]. As before, let $g=g(t)$ be a smooth solution of the Ricci flow on $M \times[0, T)$ for a closed manifold $M$ of dimension $n \geq 3$ and some $T>0$. We also state the special case $\lambda_{0}\left(g_{0}\right)>0$ as a separate theorem.

Theorem 1.5 Assume $T<\infty$ and $1<p<n$. There is a positive constant $C$ depending only on the dimension $n$, a non-positive lower bound for $R_{g_{0}}$, a positive lower bound for $\operatorname{vol}_{g_{0}}(M)$, an upper bound for $C_{S}\left(M, g_{0}\right)$, an upper bound for $T$, an upper bound for $\frac{1}{p-1}$, and an upper bound for $\frac{1}{n-p}$, such that for each $t \in[0, T)$ and all $u \in W^{1, p}(M)$ there holds

$$
\|u\|_{\frac{n p}{n-p}} \leq C\left(1+R_{\max }^{+}\right)^{\frac{1}{2}}\|u\|_{B, 1, p} .
$$

Theorem 1.6 Assume that $\lambda_{0}\left(g_{0}\right)>0$. Let $1<p<n$. There is a positive constant $C$ depending only on the dimension $n$, a positive lower bound for $\lambda_{0}\left(g_{0}\right)$, a positive lower bound for $\operatorname{vol}_{g_{0}}(M)$, an upper bound for $C_{S}\left(M, g_{0}\right)$, an upper bound for $\frac{1}{p-1}$, and an upper bound for $\frac{1}{n-p}$, such that for each $t \in[0, T)$ and all $u \in W^{1, p}(M)$ there holds

$$
\|u\|_{\frac{n p}{n-p}} \leq C\left(1+R_{\max }^{+}\right)^{\frac{1}{2}}\|u\|_{B, 1, p}
$$

The inequality (1.12) in Theorem 1.4 is a special case of the following more general result.

Theorem 1.7 Let $(M, g)$ be a metrically complete manifold, possibly with boundary. Let $\Psi \in L^{\infty}(M)$ and $\mu>1$. Assume that $\Psi \geq 0$ and the Sobolev inequality

$$
\left(\int_{M}|u|^{\frac{2 \mu}{\mu-2}} \mathrm{dvol}\right)^{\frac{\mu-2}{\mu}} \leq A \int_{M}\left(|\nabla u|^{2}+\Psi u^{2}\right) \mathrm{dvol}
$$


for some $A>0$ and all $u \in W^{1,2}(M)$. Set $H=-\Delta+\Psi$. Let $1<p<\mu$. Then there holds

$$
\left\|H^{-\frac{1}{2}} u\right\|_{\frac{\mu p}{\mu-p}} \leq C(A, \mu, p)\|u\|_{p}
$$

for all $u \in L^{p}(M)$, where the positive constant $C(\mu, c, p)$ can be bounded from above in terms of upper bounds for $A, \mu, \frac{1}{\mu-p}$ and $\frac{1}{p-1}$. Consequently, there holds for each $1<p<\infty$

$$
\|u\|_{\frac{\mu p}{\mu-p}} \leq C(A, \mu, p)\left\|H^{\frac{1}{2}} u\right\|_{p}
$$

for all $u \in W^{1, p}(M)$, provided that $(M, g)$ is compact.

\subsection{Part C}

Next we have the following consequence of Theorem 1.7.

Theorem 1.8 Let $(M, g)$ be a compact Riemannian manifold of dimension $n \geq 2$, with or without boundary. Assume $\Psi \in L^{\infty}(M)$ and $\mu>2$. Assume $\Psi \geq 0$ and the Sobolev inequality

$$
\left(\int_{M}|u|^{\frac{2 \mu}{\mu-2}} \mathrm{dvol}\right)^{\frac{\mu-2}{\mu}} \leq A \int_{M}\left(|\nabla u|^{2}+\Psi u^{2}\right) \mathrm{dvol}
$$

for all $u \in W^{1,2}(M)$. Let $1<p<\frac{\mu}{2}$. Then there holds

$$
\|u\|_{\frac{\mu p}{\mu-2 p}} \leq C(\mu, A, p)\|\Delta u+\Psi u\|_{p}
$$

for all $u \in W^{2, p}(M)$, where the constant $C(\mu, A, p)$ can be bounded from above in terms of upper bounds for $\mu, A, \frac{1}{\mu-2 p}$ and $\frac{1}{p-1}$.

Combining this theorem with the results in $[9,10]$ and [11] we then obtain $W^{2, p}$ Sobolev inequalities along the Ricci flow. Again, we only state the results in the situation of [9]. Let $g=g(t)$ be a smooth solution of the Ricci flow on $M \times[0, T)$ for a closed manifold of dimension $n \geq 3$ and some $0<T \leq \infty$, with a given initial metric $g_{0}$.

Theorem 1.9 Assume $T<\infty$ and $1<p<\frac{n}{2}$. There is a positive constant $C$ depending only on the dimension $n$, a non-positive lower bound for $R_{g_{0}}$, a positive lower bound for $\operatorname{vol}_{g_{0}}(M)$, an upper bound for $C_{S}\left(M, g_{0}\right)$, an upper bound for $T$, an upper bound for $\frac{1}{p-1}$, and an upper bound for $\frac{1}{n-2 p}$, such that for each $t \in[0, T)$ there holds

$$
\|u\|_{\frac{n p}{n-2 p}} \leq C\left\|\Delta u-\left(\frac{R}{4}-\frac{\min R_{g_{0}}^{-}}{4}+1\right) u\right\|_{p}
$$


for all $u \in W^{2, p}(M)$.

Theorem 1.10 Assume that $R_{g_{0}} \geq 0$ and $\lambda_{0}\left(g_{0}\right)>0$ (thus $R_{g_{0}}$ is somewhere positive). Let $1<p<\frac{n}{2}$. There is a positive constant $C$ depending only on the dimension $n$, a positive lower bound for $\lambda_{0}\left(g_{0}\right)$, a positive lower bound for $\operatorname{vol}_{g_{0}}(M)$, an upper bound for $C_{S}\left(M, g_{0}\right)$, an upper bound for $\frac{1}{p-1}$, and an upper bound for $\frac{1}{n-2 p}$, such that for each $t \in[0, T)$ there holds

$$
\|u\|_{\frac{n p}{n-2 p}} \leq C\left\|\Delta u-\frac{R}{4} u\right\|_{p}
$$

for all $u \in W^{2, p}(M)$.

\subsection{Part D}

Now we address the issue of converting the non-local $W^{1, p}$ Sobolev inequalities in Part B into conventional $W^{1, p}$ Sobolev inequalities. Let $H$ denote the operator $-\Delta+\Psi$. Obviously, the desired conversion requires an estimate of the following kind

$$
\left\|H^{\frac{1}{2}} u\right\|_{p} \leq C\|u\|_{1, p}
$$

for all $u \in W^{1, p}(M)$. Assume that $M$ is compact. Since $H$ is a pseudo-differential operator of order 1 [7], the inequality (1.23) holds true for some $C$, as mentioned before for the special case $\Psi=1$. But the constant $C$ obtained this way depends on $M$ and the metric $g$ in rather complicated ways. Our purpose is to obtain a constant $C$ which has clear and rudimentary geometric dependence. For this purpose, the general theory of pseudo-differential operators does not seem to give any information.

The issue at hand can be understood in terms of the Riesz transform of $H$, which is defined to be $R_{H}=\nabla H^{-\frac{1}{2}}$. An $L^{p}$ inequality for the Riesz transform

$$
\left\|R_{H} u\right\|_{p} \leq c\|u\|_{p}
$$

for all $u \in L^{p}(M)$ means the same as

$$
\|\nabla u\|_{p} \leq c\left\|H^{\frac{1}{2}} u\right\|_{p}
$$

for all $u \in W^{1, p}(M)$. On the other hand, by duality, the inequality (1.25) implies (1.23) for the dual exponent under suitable conditions on $\Psi$. (In the special case $\Psi=0$, it leads to $\left\|H^{\frac{1}{2}} u\right\|_{q} \leq c\|\nabla u\|_{q}$ for the dual exponent $q$.) In general, the Riesz transform $R_{L}$ of a non-negative symmetric elliptic operator $L$ (of second order) is defined in the same way as $R_{H}$. A fundamental problem in harmonic analysis and potential theory is to obtain $L^{p}$ boundedness for Riesz transforms $R_{L}$, or inequalities $\|\nabla u\|_{p} \leq C\left\|L^{\frac{1}{2}} u\right\|_{p}$ and $\left\|L^{\frac{1}{2}} u\right\|_{p} \leq C\|u\|_{1, p}$. From a geometric point of view, $L^{p}$ boundedness alone is not enough. It is crucial to obtain geometric estimates for the constants. 
Based on the $L^{p}$ estimates for Riesz transforms due to Bakry [1], we can convert the non-local $W^{1, p}$ Sobolev inequalities in Theorem 1.4 to conventional $W^{1, p}$ Sobolev inequalities which depend on a lower bound for the Ricci curvature. The situation of Theorem 1.7 with a general $\Psi$ is more complicated, our corresponding result will be presented elsewhere.

Theorem 1.11 Let $(M, g)$ be a complete manifold (without boundary) of dimension $n \geq 2$. Let $1<\mu<\infty$. Assume the Sobolev inequality

$$
\left(\int_{M}|u|^{\frac{2 \mu}{\mu-2}} \mathrm{dvol}\right)^{\frac{\mu-2}{\mu}} \leq A \int_{M}|\nabla u|^{2} \mathrm{dvol}+B \int_{M}|u|^{2} \mathrm{dvol}
$$

for all $u \in W^{1,2}(M)$. Assume Ric $\geq-a^{2} g$ with $a \geq 0$. Let $1<p<\mu$. Then there holds

$$
\|u\|_{\frac{\mu p}{\mu-p}} \leq C(\mu, A, B, p)\left(\|\nabla u\|_{p}+(1+a)\|u\|_{p}\right)
$$

for all $u \in W^{1, p}(M)$, where the constant $C(\mu, A, B, p)$ can be bounded from above in terms of upper bounds for $A, B, \mu, \frac{1}{\mu-p}$ and $\frac{1}{p-1}$.

We formulate the corresponding results for the Ricci flow in the situation of [9]. The results in the situations of [10] and [11] can be formulated in a similar way.

Consider a smooth solution $g=g(t)$ of the Ricci flow on $M \times[0, T)$, with initial metric $g_{0}$, where $M$ is a closed manifold of dimension $n \geq 3$. Let $\kappa=\kappa(t)$ denote $(-\min \{0, \min \operatorname{Ric}\})^{1 / 2}$ at time $t$.

Theorem 1.12 Assume $T<\infty$ and $1<p<n$. There is a positive constant $C$ depending only on the dimension $n$, a non-positive lower bound for $R_{g_{0}}$, a positive lower bound for $\operatorname{vol}_{g_{0}}(M)$, an upper bound for $C_{S}\left(M, g_{0}\right)$, an upper bound for $T$, an upper bound for $\frac{1}{p-1}$, and an upper bound for $\frac{1}{n-p}$, such that for each $t \in[0, T)$ and all $u \in W^{1, p}(M)$ there holds

$$
\|u\|_{\frac{n p}{n-p}} \leq C\left(1+R_{\max }^{+}\right)^{\frac{1}{2}}\left(\|\nabla u\|_{p}+(1+\kappa)\|u\|_{p}\right) .
$$

Theorem 1.13 Assume $\lambda_{0}\left(g_{0}\right)>0$. Let $1<p<n$. There is a positive constant $C$ depending only on the dimension $n$, a positive lower bound for $\lambda_{0}\left(g_{0}\right)$, a positive lower bound for $\operatorname{vol}_{g_{0}}(M)$, an upper bound for $C_{S}\left(M, g_{0}\right)$, an upper bound for $\frac{1}{p-1}$, and an upper bound for $\frac{1}{n-p}$, such that for each $t \in[0, T)$ and all $u \in W^{1, p}(M)$ there holds

$$
\|u\|_{\frac{n p}{n-p}} \leq C\left(1+R_{\max }^{+}\right)^{\frac{1}{2}}\left(\|\nabla u\|_{p}+(1+\kappa)\|u\|_{p}\right) .
$$

We would like to point out the obvious similarities, as well as delicate differences between these results and those in Part A. One should also compare these results and the results in Part E with Gallot's estimates of the isoperimetric constant [4,5] which 
imply estimates for the Sobolev inequalities. In contrast to Gallot's estimates, no upper bound for the diameter nor positive lower bound for the volume of $g(t)$ is assumed. Moreover, in (1.28) the lower bound for the Ricci curvature does not appear in front of $\|\nabla u\|_{p}$. (Gallot's estimates lead to Sobolev inequalities in which the lower bound for the Ricci curvature gets involved with $\|\nabla u\|_{p}$.)

\subsection{Part E}

Based on the $L^{p}$ estimates of Riesz transforms due to $\mathrm{Li}$ [5], we obtain a variant of the results in Part D in the case $1<p<2$. The non-positive lower bound for the Ricci curvature is replaced by the $L^{\frac{n}{2}+\epsilon}$ bound for the (adjusted) negative part of the Ricci curvature, where $\epsilon>0$. For a Riemannian manifold $(M, g)$ we set $\operatorname{Ric}_{\min }(x)=\min \left\{\operatorname{Ric}(v, v): v \in T_{x} M,|v|=1\right\}$.

Theorem 1.14 Let $(M, g)$ be a complete manifold (without boundaryrm) of dimension $n \geq 3$. Assume the Sobolev inequality

$$
\left(\int_{M}|u|^{\frac{2 n}{n-2}} \mathrm{dvol}\right)^{\frac{n-2}{n}} \leq A \int_{M}|\nabla u|^{2} \mathrm{dvol}+B \int_{M}|u|^{2} \mathrm{dvol}
$$

for all $u \in W^{1,2}(M)$. Let $c \geq 0$ and $\epsilon>0$ and assume $\left(\operatorname{Ric}_{\min }+c\right)^{-} \in L^{\frac{n}{2}+\epsilon}(M)$. Let $1<p<2$. Then there holds

$$
\|u\|_{\frac{n p}{n-p}} \leq C\left(\|\nabla u\|_{p}+(1+\gamma)\|u\|_{p}\right)
$$

for all $u \in W^{1, p}(M)$, where

$$
\gamma=\left(\int_{M}\left|\left(\mathrm{Ric}_{\min }+c\right)^{-}\right|^{\frac{n}{2}+\epsilon} \mathrm{dvol}\right)^{\frac{1}{2 \epsilon}}
$$

and the constant $C$ can be bounded from above in terms of upper bounds for $n, A, B$, $c, \frac{1}{\epsilon}$ and $\frac{1}{p-1}$.

Next we consider a smooth solution $g=g(t)$ of the Ricci flow on $M \times[0, T)$ for a closed manifold $M$ of dimension $n \geq 3$ and $T>0$.

Theorem 1.15 Assume $T<\infty$. Let $\epsilon>0$ and $1<p<2$. There is a positive constant $C$ depending only on the dimension $n$, a non-positive lower bound for $R_{g_{0}}, a$ positive lower bound for $\operatorname{vol}_{g_{0}}(M)$, an upper bound for $C_{S}\left(M, g_{0}\right)$, an upper bound for $T$, an upper bound for $\frac{1}{p-1}$, and an upper bound for $\frac{1}{\epsilon}$, such that for each $t \in[0, T)$ and all $u \in W^{1, p}(M)$ there holds

$$
\|u\|_{\frac{n p}{n-p}} \leq C\left(1+R_{\max }^{+}\right)^{\frac{1}{2}}\left(\|\nabla u\|_{p}+(1+\gamma)\|u\|_{p}\right),
$$

where $\gamma=\gamma(t)$ is the same quantity as in Theorem 1.14. 
Theorem 1.16 Assume $\lambda_{0}\left(g_{0}\right)>0$. Let $\epsilon>0$ and $1<p<2$. There is a positive constant $C$ depending only on the dimension $n$, a positive lower bound for $\lambda_{0}\left(g_{0}\right), a$ positive lower bound for $\operatorname{vol}_{g_{0}}(M)$, an upper bound for $C_{S}\left(M, g_{0}\right)$, an upper bound for $\frac{1}{p-1}$ and an upper bound for $\frac{1}{\epsilon}$, such that for each $t \in[0, T)$ and all $u \in W^{1, p}(M)$ there holds

$$
\|u\|_{\frac{n p}{n-p}} \leq C\left(1+R_{\max }^{+}\right)^{\frac{1}{2}}\left(\|\nabla u\|_{p}+(1+\gamma)\|u\|_{p}\right),
$$

where

$$
\gamma=\gamma(t)=\left(\int_{M} \mid\left(\operatorname{Ric}_{\min }-\frac{1}{n} \min R_{g_{0}}^{-}\right)^{-\left.\right|^{\frac{n}{2}+\epsilon} \mathrm{dvol}}\right)^{\frac{1}{2 \epsilon}} .
$$

\section{From $W^{1, p}$ for Lower $p$ to $W^{1, p}$ for Higher $p$}

Theorem 2.1 Consider a Riemannian manifold $(M, g)$ of dimension $n \geq 2$, with or without boundary. Let $1 \leq p_{0}<n$. Assume that the Sobolev inequality

$$
\left(\int_{M}|u|^{\frac{n p_{0}}{n-p_{0}}} \mathrm{dvol}\right)^{\frac{n-p_{0}}{n}} \leq A \int_{M}|\nabla u|^{p_{0}} \mathrm{dvol}+\frac{B}{\operatorname{vol}_{g}(M)^{\frac{2}{n}}} \int_{M}|u|^{p_{0}} \mathrm{dvol}
$$

holds true for all $u \in W^{1, p_{0}}(M)$ with some $A>0$ and $B>0$. Then we have

$$
\left(\int_{M}|u|^{\frac{p n}{n-p}}\right)^{\frac{n-p}{n}} \leq 2^{\frac{p-p_{0}}{p_{0}}} A^{\frac{p}{p_{0}}}\left(r(p)^{p_{0}}+B\right)^{\frac{p}{p_{0}}} \int_{M}|\nabla u|^{p}+\frac{2^{\frac{p-p_{0}}{p_{0}}} B^{\frac{2 p}{p_{0}}}}{\operatorname{vol}_{g}(M)^{\frac{p}{n}}} \int_{M} u^{p}
$$

for each $p_{0}<p \leq \frac{n^{2} p_{0}}{\left(n-p_{0}\right)^{2}+n p_{0}}$ and all $u \in W^{1, p}(M)$, where $r(p)=\frac{p\left(n-p_{0}\right)}{p_{0}(n-p)}$ and the notation of the volume form is omitted. If $\operatorname{vol}_{g}(M)=\infty$, it is understood that the second term on the right hand side in (2.1) and (2.2) is zero, and $B$ is absent elsewhere.

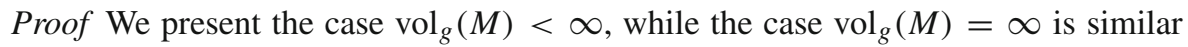
and much easier. By scaling invariance we can assume $\operatorname{vol}_{g}(M)=1$. Consider $u \in$ $C_{c}^{\infty}(M)$ and set $v=|u|^{r}$ for $r>1$. Then we have

$$
\left(\int_{M}|u|^{\frac{n p_{0} r}{n-p_{0}}}\right)^{\frac{n-p_{0}}{n}} \leq A r^{p_{0}} \int_{M}|u|^{p_{0}(r-1)}|\nabla u|^{p_{0}}+B \int_{M}|u|^{p_{0} r} .
$$


For a given $p_{0}<p<n$ we choose $r=r_{p}$ and hence $r_{p}-1=\frac{n\left(p-p_{0}\right)}{p_{0}(n-p)}$. Then we infer by Hölder's inequality

$$
\begin{aligned}
\left(\int_{M}|u|^{\frac{n p}{n-p}}\right)^{\frac{n-p_{0}}{n}} & \leq A r_{p}^{p_{0}} \int_{M}|u|^{\frac{n\left(p-p_{0}\right)}{n-p}}|\nabla u|^{p_{0}}+B \int_{M}|u|^{\frac{p\left(n-p_{0}\right)}{n-p}} \\
& \leq A r_{p}^{p_{0}}\left(\int_{M}|u|^{\frac{n p}{n-p}}\right)^{\frac{p-p_{0}}{p}} \cdot\left(\int_{M}|\nabla u|^{p}\right)^{\frac{p_{0}}{p}}+B \int_{M}|u|^{\frac{p\left(n-p_{0}\right)}{n-p}} .
\end{aligned}
$$

Now we assume $p_{0}<p \leq \frac{n^{2} p_{0}}{\left(n-p_{0}\right)^{2}+n p_{0}}$. Then $\frac{p\left(n-p_{0}\right)}{n-p} \leq \frac{n p_{0}}{\left.n-p_{0}\right)}$. Since $\operatorname{vol}_{g}(M)=$ 1 we have by Hölder's inequality

$$
\int_{M}|u|^{\frac{p\left(n-p_{0}\right)}{n-p}} \leq\left(\int_{M}|u|^{\frac{n p_{0}}{n-p_{0}}}\right)^{\frac{p\left(n-p_{0}\right)^{2}}{n p_{0}(n-p)}}
$$

and

$$
\int_{M}|u|^{\frac{n p_{0}}{n-p_{0}}} \leq\left(\int_{M}|u|^{\frac{n p}{n-p}}\right)^{\frac{p_{0}(n-p)}{\left(n-p_{0}\right) p}}
$$

We deduce

$$
\begin{aligned}
\left(\int_{M}|u|^{\frac{p n}{n-p}}\right)^{\frac{\left(n-p_{0}\right)}{n}-\frac{p-p_{0}}{p}} \leq & A r_{p}^{p_{0}}\left(\int_{M}|\nabla u|^{p}\right)^{\frac{p_{0}}{p}} \\
& +B\left(\int_{M}|u|^{\frac{n p_{0}}{n-p_{0}}}\right)^{\frac{p\left(n-p_{0}\right)^{2}}{n p_{0}(n-p)}-\frac{\left(n-p_{0}\right)\left(p-p_{0}\right)}{p_{0}(n-p)}}
\end{aligned}
$$

which leads to

$$
\begin{aligned}
\left(\int_{M}|u|^{\frac{p n}{n-p}}\right)^{\frac{p_{0}(n-p)}{n p}} & \leq A r_{p}^{p_{0}}\left(\int_{M}|\nabla u|^{p}\right)^{\frac{p_{0}}{p}}+B\left(\int_{M}|u|^{\frac{n p_{0}}{n-p_{0}}}\right)^{\frac{n-p_{0}}{n}} \\
& \leq A r_{p}^{p_{0}}\left(\int_{M}|\nabla u|^{p}\right)^{\frac{p_{0}}{p}}+A B \int_{M}|\nabla u|^{p_{0}}+B^{2} \int_{M} u^{p_{0}} \\
& \leq A\left(r_{p}^{p_{0}}+B\right)\left(\int_{M}|\nabla u|^{p}\right)^{\frac{p_{0}}{p}}+B^{2}\left(\int_{M} u^{p}\right)^{\frac{p_{0}}{p}}
\end{aligned}
$$

It follows that

$$
\left(\int_{M}|u|^{\frac{p n}{n-p}}\right)^{\frac{n-p}{n}} \leq 2^{\frac{p-p_{0}}{p_{0}}} A^{\frac{p}{p_{0}}}\left(r_{p}^{p_{0}}+B\right)^{\frac{p}{p_{0}}} \int_{M}|\nabla u|^{p}+2^{\frac{p-p_{0}}{p_{0}}} B^{\frac{2 p}{p_{0}}} \int_{M} u^{p}
$$


By approximation, this holds for all $u \in W^{1, p}(M)$.

Remark We can also consider the following assumption

$$
\left(\int_{M}|u|^{\frac{n p_{0}}{n-p_{0}}} \mathrm{dvol}\right)^{\frac{n-p_{0}}{n}} \leq A \int_{M}|\nabla u|^{p_{0}} \mathrm{dvol}+\int_{M} f|u|^{p_{0}} \mathrm{dvol}
$$

with a given function $f$. It is easy to adapt the above proof to obtain Sobolev inequalities for higher $p$ in terms of an $L^{q}$ bound of $f$ for a suitable $q$. This can be applied to the Ricci flow to yield Sobolev inequalities in terms of an $L^{q}$ bound of the scalar curvature.

Lemma 2.2 Let $1 \leq p_{0}<n$. We set $p_{k+1}=\frac{n^{2} p_{k}}{\left(n-p_{k}\right)^{2}+n p_{k}}$ for $k \geq 0$. Then $1 \leq p_{k}<n$ for all $k$. Moreover, the sequence $p_{k}$ is increasing and converges to $n$.

Proof The inequality $p_{k+1}<n$ is equivalent to $\left(n-p_{k}\right)^{2}>0$, while the inequality $p_{k} \geq 1$ is equivalent to $\left(n^{2}+n\right) p_{k}+p_{k}^{2} \geq n^{2}$. Hence $1 \leq p_{k}<n$ follows from the induction. Since $p_{k}<n$, we have $\left(n-p_{k}\right)^{2}+n p_{k}<n^{2}$, and hence $p_{k+1}>p_{k}$. Let $p_{*}$ denote the limit of $p_{k}$. Then $p_{*}=\frac{n^{2} p_{*}}{\left(n-p_{*}\right)^{2}+n p_{*}}$. It follows that $p_{*}=n$.

Proof of Theorem 1.1 Applying Theorem 2.1 repeatedly, starting with $p_{0}=2$. By induction and Lemma 2.2, we then arrive at the desired Sobolev inequalities.

Proof of Theorem 1.3 We first observe the following property of the inequality (1.5): if $A=\alpha A_{1}$ and $B=\alpha B_{1}$ for some $\alpha \geq 1$, then we have

$$
C_{1}\left(n, p_{0}, p, A, B\right) \leq \alpha^{\frac{m(p) p}{p_{0}}} C_{1}\left(n, p_{0}, p, A_{1}, B_{1}\right)
$$

and

$$
C_{2}\left(n, p_{0}, p, A, B\right) \leq \alpha^{\frac{m(p) p}{p_{0}}} C_{2}\left(n, p_{0}, p, A_{1}, B_{1}\right)
$$

where $m(p)=2^{k+1}$ for $p \in\left(p_{k}, p_{k+1}\right]$ (see Lemma 2.2 for $\left.p_{k}\right)$. This follows from the formula (2.2). By [Theorem D*, 12], the Sobolev inequality (1.2) holds true, where $A$ has the dependence as stated in Theorem 1.3, without reference to $p$. We then have

$$
\left(\int_{M}|u|^{\frac{2 n}{n-2}} \mathrm{dvol}\right)^{\frac{n-2}{n}} \leq A\left(1+\max R^{+} \operatorname{vol}(M)^{\frac{2}{n}}\right) \int_{M}\left(|\nabla u|^{2}+\frac{u^{2}}{\operatorname{vol}(M)^{\frac{2}{n}}}\right) \mathrm{dvol} .
$$

Applying Theorem 1.1 and the above observation we then arrive at the desired Sobolev inequality.

Theorem 1.2 can be proved in the same way. 


\section{Non-local Sobolev Inequalities in Terms of the $(1, p)$-Bessel Norm}

First we extend the general results in [9] on the heat semi-group and the non-local Sobolev inequalities to general metrically complete manifolds with or without boundary. Consider a Riemannian manifold $(M, g)$ of dimension $n \geq 2$, and a function $\Psi \in L^{\infty}(M)$. We set as in [9] $H=-\Delta+\Psi$ and $Q(u)=\int_{M}\left(|\nabla u|^{2}+\Psi u^{2}\right)$ dvol.

Theorem 3.1 Let $(M, g)$ be metrically complete manifold possibly with boundary. Let $0<\sigma^{*} \leq \infty$. Assume that for each $0<\sigma<\sigma^{*}$ the logarithmic Sobolev inequality

$$
\int_{M} u^{2} \ln u^{2} \mathrm{dvol} \leq \sigma Q(u)+\beta(\sigma)
$$

holds true for all $u \in W^{1,2}(M)$ with $\|u\|_{2}=1$, where $\beta$ is a non-increasing continuous function. Assume that

$$
\tau(t)=\frac{1}{2 t} \int_{0}^{t} \beta(\sigma) d \sigma
$$

is finite for all $0<t<\sigma^{*}$. Then there holds

$$
\left\|e^{-t H} u\right\|_{\infty} \leq e^{\tau(t)-\frac{3 t}{4} \inf \Psi^{-}}\|u\|_{2}
$$

for each $0<t<\frac{1}{4} \sigma^{*}$ and all $u \in L^{2}(M)$. There also holds

$$
\left\|e^{-t H} u\right\|_{\infty} \leq e^{2 \tau\left(\frac{t}{2}\right)-\frac{3 t}{4} \inf \Psi^{-}}\|u\|_{1}
$$

for each $0<t<\frac{1}{4} \sigma^{*}$ and all $u \in L^{1}(M)$.

Theorem 3.2 Let $(M, g)$ be a metrically complete manifold, possibly with boundary.

1) Let $\mu>1$. Assume that $\Psi \geq 0$ and for some $c>0$ the inequality

$$
\left\|e^{-t H} u\right\|_{\infty} \leq c t^{-\frac{\mu}{4}}\|u\|_{2}
$$

holds true for each $t>0$ and all $u \in L^{2}(M)$. Let $1<p<\mu$. Then there holds

$$
\left\|H^{-\frac{1}{2}} u\right\|_{\frac{\mu p}{\mu-p}} \leq C(c, \mu, p)\|u\|_{p}
$$

for all $u \in L^{p}(M)$, where the positive constant $C(\mu, c, p)$ can be bounded from above in terms of upper bounds for $c, \mu, \frac{1}{\mu-p}$ and $\frac{1}{p-1}$. Consequently, there holds

$$
\|u\|_{\frac{2 \mu}{\mu-2}} \leq C(c, 2, p)\left(\int_{M}\left(|\nabla u|^{2}+\Psi u^{2}\right) \mathrm{dvol}\right)^{\frac{1}{2}}
$$


for all $u \in W^{1,2}(M)$. Moreover, there holds for a given $1<p<\infty$

$$
\|u\|_{\frac{\mu p}{\mu-p}} \leq C(c, \mu, p)\left\|H^{\frac{1}{2}} u\right\|_{p}
$$

for all $u \in W^{1, p}(M)$, provided that $(M, g)$ is $p$-Bessel.

2) Let $\mu>1$. Assume that for some $c>0$ the inequality

$$
\left\|e^{-t H} u\right\|_{\infty} \leq c t^{-\frac{\mu}{4}}\|u\|_{2}
$$

holds true for each $0<t<1$ and all $u \in L^{2}(M)$. Set $H_{0}=H-\inf \Psi^{-}+1$. Let $1<p<\mu$. Then there holds

$$
\left\|H_{0}^{-\frac{1}{2}} u\right\|_{\frac{\mu p}{\mu-p}} \leq C(\mu, c, p)\|u\|_{p}
$$

for all $u \in L^{p}(M)$, where the positive constant $C(\mu, c, p)$ has the same property as the $C(\mu, c, p)$ above. Consequently, there holds

$$
\|u\|_{\frac{2 \mu}{\mu-2}} \leq C(\mu, 2, p)\left(\int_{M}\left(|\nabla u|^{2}+\left(\Psi-\inf \Psi^{-}+1\right) u^{2}\right) \mathrm{dvol}\right)^{\frac{1}{2}}
$$

for all $u \in W^{1,2}(M)$. Moreover, there holds for a given $1<p<\infty$

$$
\|u\|_{\frac{\mu p}{\mu-p}} \leq C(\mu, c, p)\left\|H_{0}^{\frac{1}{2}} u\right\|_{p}
$$

for all $u \in W^{1, p}(M)$, provided that $(M, g)$ is p-Bessel.

To establish these two results, we need the following two ingredients: the construction of the heat semi-group $\mathrm{e}^{-t H}$ and the $L^{p}$ contraction properties of $\mathrm{e}^{-t H}$ for all $1<p<\infty$. In [9], since the manifold is assumed to be closed, the heat semi-group is constructed by the spectral representation in terms of the eigenfunctions. This works equally well on a compact manifold with boundary, where the eigenfunctions satisfy the Neumann boundary condition. For a general metrically complete manifold, we follow the construction in [8] based on the general theory of spectral representation of self-adjoint operators. The case of $H=-\Delta$ on a complete manifold without boundary is treated in [8], but the arguments extend to general metrically complete manifolds and $H=-\Delta+\Psi$ with $\Psi \in L^{\infty}(M)$ and $\Psi \geq 0$, as explained below.

Consider $\Psi \in L^{\infty}(M)$ with $\Psi \geq 0$. The initial domain for $H=-\Delta+\Psi$ is the space $\Omega_{H}=C_{c, N}^{\infty}(M)=\left\{u \in C_{c}^{\infty}(M): \frac{\partial u}{\partial v}=0\right\}$, where $v$ denotes the inward unit normal of $\partial M$. Note that $\Omega_{H}$ is dense in $L^{2}(M)$. Let $H_{\text {min }}$ denote the $L^{2}$ closure of $H$, whose domain $D\left(H_{\min }\right)$ consists of all $u \in L^{2}(M)$ such that there is a sequence $u_{i} \in \Omega_{H}$ such that $u_{i} \rightarrow u$ in $L^{2}(M)$ and $H u_{i}$ converges in $L^{2}(M)$ to some function, which we can write $H u$. Let $H_{\max }$ be the adjoint of $H_{\min }$ in $L^{2}(M)$, and $D\left(H_{\max }\right) \subset L^{2}(M)$ its domain. We have the following extension of [8, Lemma 2.3]. 
Lemma 3.3 Let $(M, g)$ be metrically complete. Assume that $u \in D\left(H_{\max }\right)$ satisfies $H u=\lambda u$ for some $\lambda<0$. Then $u \equiv 0$.

Proof By basic elliptic regularity, we have $u \in W_{l o c}^{2, p}(M)$ for all $p>0$ and $\frac{\partial u}{\partial v}=0$. ( By the Sobolev embedding we have $u \in C^{1}(M)$. ) Fix $x_{0} \in M$ and let $\varphi(x)=$ $\varphi_{r_{1}, r_{2}}(x)=\psi\left(\left(r_{2}-r_{1}\right)^{-1}\left(d\left(x_{0}, x\right)+r_{2}-2 r_{1}\right)\right)$ for a smooth function $\psi(t)$ which is 1 for $t \leq 1$ and 0 for $t \geq 2$. Then $\varphi(x)=1$ if $d\left(x_{0}, x\right) \leq r_{1}, \varphi(x)=0$ if $d\left(x_{0}, x\right) \geq r_{2}$, and $|\nabla \varphi| \leq c\left(r_{2}-r_{1}\right)^{-1}$ for a constant $c$.

Now we have

$$
\begin{aligned}
\lambda\left\langle\varphi^{2} u, u\right\rangle_{2} & =\left\langle\varphi^{2} u, H u\right\rangle_{2}=-\left\langle\varphi^{2} u, \Delta u\right\rangle_{2}+\left\langle\varphi^{2} u, \Psi u\right\rangle \\
& \geq-\left\langle\varphi^{2} u, \Delta u\right\rangle_{2}=\left\|\varphi^{2} \nabla u\right\|_{2}^{2}+2\langle u \nabla \phi, \phi \nabla u\rangle_{2} .
\end{aligned}
$$

It follows that

$$
\left\|\varphi^{2} \nabla u\right\|_{2}^{2} \leq \lambda\left\langle\varphi^{2} u, u\right\rangle_{2}+2\langle u \nabla \phi, \phi \nabla u\rangle_{2} .
$$

By Schwarz inequality we then deduce

$$
\left\|\varphi^{2} \nabla u\right\|_{2}^{2} \leq 2 \lambda\left\langle\varphi^{2} u, u\right\rangle_{2}+\frac{4 c^{2}}{\left(r_{2}-r_{1}\right)^{2}}\|u\|_{2}^{2} .
$$

Letting first $r_{2} \rightarrow \infty$ and then $r_{1} \rightarrow \infty$ we arrive at

$$
\|\nabla u\|_{2}^{2} \leq 2 \lambda\|u\|_{2}^{2}
$$

Since $\lambda<0$, we conclude $u \equiv 0$.

By this lemma and [8, Lemma 2.1] we infer that $H_{\max }=H_{\min }$, which is the self-adjoint extension of $H$. Now we can apply the spectral theorem for self-adjoint operators to obtain the heat semi-group $\mathrm{e}^{-t H}$ and other potentials of $H$ such as $H^{-\frac{1}{2}}$ and $H^{\frac{1}{2}}$.

In [9], the $L^{p}$ contraction property of $\mathrm{e}^{-t H}$ is derived in terms of the $L^{2}$ contraction property and the $L^{\infty}$ contraction property, with the latter implied by the maximum principle. This argument can be extended to compact manifolds with boundary. But the maximum principle may not hold on general complete, non-compact manifolds. Instead, we follow the arguments in [8] for obtaining the $L^{p}$ contraction property. By the arguments in Sect. 3 of [8], in order to show that $\mathrm{e}^{-t H}$ is a contraction on $L^{p}(M) \cap L^{2}(M)$ for each $1 \leq p \leq \infty$, it suffices to establish the following two lemmas.

Lemma 3.4 For each $1<p<\infty$ the operator $H$ with domain $\Omega_{H}$ is dissipative, i.e., for each nonzero $u \in \Omega_{H}$, there is a function $v \in L^{q}$ with $q=\frac{p}{p-1}$ such that $\|v\|_{q}=\|u\|_{p},\langle u, v\rangle_{2}=\|u\|_{p}$ and $\langle H u, v\rangle_{2} \leq 0$. 
Lemma 3.5 Let $1<p \leq q<\infty$. Assume that $u \in L^{p}(M) \cap L^{q}(M)$ satisfies $H u=\lambda u$ for some $\lambda<0$ (this contains the assumption that $u$ lies in the domain of the closure of $H$ in $L^{p}(M)$ and that in $L^{q}(M)$.) Then $u \equiv 0$.

Since $\Psi \geq 0$, the proofs of these two lemmas in [8] (for the special case that $M$ has no boundary and $\Psi=0$ ) can easily be adapted to our situation. This is similar to the above proof of Lemma 3.3.

Having established the desired construction of $\mathrm{e}^{-t H}$ and the $L^{p}$ contraction properties we make two more remarks. First, the construction of the heat semi-group $\mathrm{e}^{-t H}$ for a general $\Psi \in L^{\infty}(M)$ follows via the formula $\mathrm{e}^{-t H}=e^{-t \inf \Psi^{-}} e^{-t H_{1}}$, where $H_{1}=-\Delta+\Psi-\inf \Psi^{-}$. Second, in [9], the space $L^{\infty}(M)$ is used in the formulations of the Marcinkiewicz interpolation theorem and the Riesz-Thorin interpolation theorem. In the case of a general Riemannian manifold, we replace $L^{\infty}(M)$ by $L^{\infty}(M) \cap L^{1}(M)$.

Proof of Theorem 3.1 Consider $u_{0} \in L^{2}(M)$. We claim that $\mathrm{e}^{-t H} u_{0} \in W^{1,2}(M)$ for $t>0$. Indeed we have for $u=e^{-t H} u_{0}$

$$
\frac{\partial u}{\partial t}=H u, \quad \frac{\partial u}{\partial v}=0
$$

Then we have

$$
\frac{d}{d t} \int_{M} \varphi^{2} u^{2}=2 \int_{M} \varphi^{2} u H u=-2 \int_{M} \varphi^{2}|\nabla u|^{2}-2 \int_{M} \varphi u \nabla u \cdot \nabla \varphi-2 \int_{M} \Psi \varphi^{2} u^{2}
$$

where $\varphi=\varphi_{r_{2}, r_{1}}$ is the function in the proof of Lemma 3.3. It follows that

$$
\frac{d}{d t} \int_{M} \varphi^{2} u^{2} \leq-\int_{M} \varphi^{2}|\nabla u|^{2}+\int_{M} u^{2}|\nabla \varphi|^{2}-\int_{M} \Psi \varphi^{2} u^{2}
$$

and then

$$
\int_{M} \varphi^{2} u^{2}+\int_{0}^{t} \int_{M} \varphi^{2}|\nabla u|^{2} \leq\left.\int_{M} \varphi^{2} u^{2}\right|_{t=0}+\int_{0}^{t} \int_{M}\left(|\nabla \varphi|^{2}-\Psi\right) u^{2} .
$$

Letting $r_{2} \rightarrow \infty$ and then $r_{1} \rightarrow \infty$ we arrive at

$$
\int_{M} u^{2}+\int_{0}^{t} \int_{M}|\nabla u|^{2} \leq\left.\int_{M} u^{2}\right|_{t=0}-\int_{0}^{t} \int_{M} \Psi u^{2}
$$

It follows that $\int_{M}|\nabla u(\cdot, t)|^{2}<\infty$ for a.e. $t>0$. By continuity, $\int_{M}|\nabla u(\cdot, t)|^{2}<\infty$ for all $t>0$. Hence $\mathrm{e}^{-t H} u_{0} \in W^{1,2}(M)$ for all $t>0$.

Now we can carry over the proof of Theorem 5.3 in [9]. Some modification is necessary because $M$ is possibly non-compact. Let $\varphi=\varphi_{r_{1}, r_{2}}$ be the function in the 
proof of Lemma 3.3. In place of [9, (B.13)] we have now for $u_{s}=e^{-s H} u_{0}$ for a given $u_{0} \in W^{1,2}(M) \cap L^{\infty}(M)$

$$
\begin{aligned}
\frac{d}{d s} & \ln \left(e^{-N(s)}\left\|\varphi u_{s}\right\|_{p(s)}\right)=\frac{d}{d s}\left(-N(s)+\frac{1}{p(s)} \ln \left\|\varphi u_{s}\right\|_{p(s)}^{p(s)}\right) \\
= & \frac{\Gamma}{\sigma}-\frac{1}{p^{2}} \frac{p}{\sigma} \ln \left\|\varphi u_{s}\right\|_{p}^{p}+\frac{1}{p}\left\|\varphi u_{s}\right\|_{p}^{-p}\left(-p Q\left(\varphi u_{s}, u_{s}^{p-1}\right)+\frac{p}{\sigma} \int_{M} \varphi u_{s}^{p} \ln u_{s}\right) \\
= & \frac{1}{\sigma}\left\|\varphi u_{s}\right\|_{p}^{-p}\left(\int_{M} \varphi u_{s}^{p} \ln u_{s}-\sigma Q\left(\varphi u_{s}, u_{s}^{p-1}\right)\right. \\
& \left.-\Gamma\left\|\varphi u_{s}\right\|_{p}^{p}-\left\|\varphi u_{s}\right\|_{p}^{p} \ln \left\|\varphi u_{s}\right\|_{p}\right) .
\end{aligned}
$$

It follows that

$$
\begin{aligned}
& \ln \left(e^{-N\left(t_{2}\right)}\left\|\varphi u_{t_{2}}\right\|_{p\left(t_{2}\right)}\right) \leq \ln \left(e^{-N\left(t_{1}\right)}\left\|\varphi u_{t_{1}}\right\|_{p\left(t_{1}\right)}\right) \\
& +\int_{t_{1}}^{t_{2}} \frac{1}{\sigma}\left\|\varphi u_{s}\right\|_{p}^{-p}\left(\int_{M} \varphi u_{s}^{p} \ln u_{s}\right. \\
& \left.-\sigma Q\left(\varphi u_{s}, u_{s}^{p-1}\right)-\Gamma\left\|\varphi u_{s}\right\|_{p}^{p}-\left\|\varphi u_{s}\right\|_{p}^{p} \ln \left\|\varphi u_{s}\right\|_{p}\right) d s
\end{aligned}
$$

for $t_{2}>t_{1}>0$. Letting first $r_{2} \rightarrow \infty$ and then $r_{1} \rightarrow \infty$ we then arrive at (3.23) without the presence of $\phi$.

Proof of Theorem 3.2 Given the $L^{p}$ contraction property established above, the proof of Theorem C.5 in [9] carries over straightforwardly. The Sobolev inequality (3.7) follows because of the identity $\left\langle H^{\frac{1}{2}} u, H^{\frac{1}{2}} u\right\rangle_{2}=\int_{M}\left(|\nabla u|^{2}+\Psi u^{2}\right)$ dvol for all $u \in C_{c}^{\infty}(M)$ (then also for $u \in W^{1,2}(M)$ ). The Sobolev inequality (3.11) follows in the same fashion.

Proof of Theorem 1.7 Assume $\int_{M} u^{2}=1$. As in the proof of Theorem 3.1 in [9] we have

$$
\ln \int_{M}|u|^{\frac{2 \mu}{\mu-2}}=\ln \int_{M} u^{2}|u|^{\frac{4}{\mu-2}} \geq \int_{M} u^{2} \ln |u|^{\frac{4}{\mu-2}} .
$$

It follows that

$$
\begin{aligned}
\int_{M} u^{2} \ln u^{2} & \leq \frac{\mu}{2} \ln \left(\int_{M}|u|^{\frac{2 \mu}{\mu-2}}\right)^{\frac{\mu-2}{\mu}} \leq \frac{\mu}{2} \ln \left(A \int_{M}\left(|\nabla u|^{2}+\Psi \int_{M} u^{2}\right)\right. \\
& \leq \frac{\mu}{2} \ln A+\frac{\mu}{2} \ln \int_{M}\left(|\nabla u|^{2}+\Psi u^{2}\right) .
\end{aligned}
$$

By [9, Lemma 3.2] we then deduce each $\sigma>0$

$$
\int_{M} u^{2} \ln u^{2} \leq \frac{\mu}{2} \sigma \int_{M}\left(|\nabla u|^{2}+\Psi u^{2}\right)-\frac{\mu}{2} \ln \sigma+\frac{\mu}{2} \ln A-1,
$$


which leads to

$$
\int_{M} u^{2} \ln u^{2} \leq \sigma \int_{M}\left(|\nabla u|^{2}+\Psi u^{2}\right)-\frac{\mu}{2} \ln \sigma+\frac{\mu}{2} \ln \frac{\mu}{2}+\frac{\mu}{2} \ln A-1 .
$$

By Theorem 3.1 we deduce for $H=-\Delta+1$

$$
\left\|e^{-t H} u\right\|_{\infty} \leq t^{-\frac{\mu}{4}} e^{\frac{\mu}{4}+\frac{A_{0}}{2}}\|u\|_{2}
$$

for all $t>0$, where $A_{0}=\frac{\mu}{2} \ln \frac{\mu}{2}+\frac{\mu}{2} \ln A-1$. Applying Theorem 3.2, we then arrive at the desired inequality (1.17).

Proof of Theorem 1.4 The Sobolev inequality (1.11) leads to

$$
\left(\int_{M}|u|^{\frac{2 \mu}{\mu-2}} \mathrm{dvol}\right)^{\frac{\mu-2}{\mu}} \leq \max \{A, B\} \int_{M}\left(|\nabla u|^{2}+u^{2}\right) \mathrm{dvol} .
$$

Hence we can apply Theorem 1.7.

Lemma 3.6 Let $\bar{g}=\lambda^{2} g$ for some $\lambda \geq 1$. Let $\mu>1$ and $1 \leq p<\mu$. Assume the inequality

$$
\|u\|_{\frac{\mu p}{\mu-p}} \leq C\|u\|_{B, 1, p}
$$

for all $u \in W^{1, p}(M)$ with respect to $\bar{g}$. Then there holds

$$
\|u\|_{\frac{\mu p}{\mu-p}} \leq \lambda C\|u\|_{B, 1, p}
$$

for all $u \in W^{1, p}(M)$ with respect to $g$.

Proof We compute the scaling change of $(-\Delta+1)^{\frac{1}{2}}$. We have $\Delta_{\bar{g}}=\lambda^{-2} \Delta$. Hence

$$
-\Delta_{\bar{g}}+1=-\lambda^{-2} \Delta+1=\lambda^{-2}\left(-\Delta+\lambda^{2}\right) .
$$

By [1, Lemma 4.2], we have for $u \in L^{p}(M)$ and $a \geq 0$

$$
c_{1}\left(a\|u\|_{p}+\left\|(-\Delta)^{\frac{1}{2}} u\right\|_{p}\right) \leq\left\|\left(-\Delta+a^{2}\right)^{\frac{1}{2}} u\right\|_{p} \leq c_{2}\left(a\|u\|_{p}+\left\|(-\Delta)^{\frac{1}{2}} u\right\|_{p}\right),
$$

where $c_{1}$ and $c_{2}$ are universal constants. It follows that

$$
\begin{aligned}
\left\|\left(-\Delta+\lambda^{2}\right)^{\frac{1}{2}} u\right\|_{p} & \leq c_{2}\left(\lambda\|u\|_{p}+\left\|(-\Delta)^{\frac{1}{2}} u\right\|_{p}\right) \\
& \leq c_{2} \lambda\left(\|u\|_{p}+\left\|(-\Delta)^{\frac{1}{2}} u\right\|_{p}\right) \\
& \leq c_{1} c_{2} \lambda\left\|(-\Delta+1)^{\frac{1}{2}} u\right\|_{p} .
\end{aligned}
$$


Hence

$$
\left\|\left(-\Delta_{\bar{g}}+1\right)^{\frac{1}{2}} u\right\|_{p} \leq c_{1} c_{2}\left\|(-\Delta+1)^{\frac{1}{2}} u\right\|_{p} .
$$

Now we have

$$
\|u\|_{\frac{p n}{n-p}, \bar{g}}=\|u\|_{\frac{n p}{n-p}} \lambda^{\frac{n-p}{p}}
$$

and

$$
\left\|\left(-\Delta_{\bar{g}}+1\right)^{\frac{1}{2}} u\right\|_{p, \bar{g}}=\left\|\left(-\Delta_{\bar{g}}+1\right)^{\frac{1}{2}} u\right\|_{p} \lambda^{\frac{n}{p}} .
$$

We arrive at

$$
\|u\|_{\frac{n p}{n-p}} \leq \lambda C\left\|(-\Delta+1)^{\frac{1}{2}} u\right\|_{p}
$$

Proof of Theorem 1.5 By [9, Theorem D], the Sobolev inequality (1.3) holds true, where $A$ and $B$ have the same property as the $C$ in the theorem, without the reference to $p$. Let $\lambda$ and $\bar{g}$ be the same as above. Then we have for $\bar{g}$

$$
\begin{aligned}
\left(\int_{M}|u|^{\frac{2 n}{n-2}} \mathrm{dvol}\right)^{\frac{n-2}{n}} & \leq A \int_{M}\left(|\nabla u|^{2}+\frac{R}{4} u^{2}\right) \mathrm{dvol}+\frac{B}{\lambda^{2}} \int_{M} u^{2} \mathrm{dvol} \\
& \leq A \int_{M}|\nabla u|^{2} \mathrm{dvol}+\left(\frac{A}{4}+B\right) \int_{M} u^{2} \mathrm{dvol} \\
& \leq(A+B) \int_{M}\left(|\nabla u|^{2}+u^{2}\right) \mathrm{dvol} .
\end{aligned}
$$

Applying Theorem 1.4 and Lemma 3.6, we arrive at the desired inequality (1.21).

Proof of Theorem 1.6 By [9, Theorem $\mathrm{D}^{*}$ ], the Sobolev inequality (1.2) holds true, where $A$ has the same property as the $C$ in the theorem without the reference to $p$. Set $\lambda=\lambda(t)=\left(1+R_{\max }^{+}\right)^{1 / 2}$ at time $t$. Then the Sobolev inequality (1.2) still holds true for $\bar{g}=\lambda^{2} g$. Since $R_{\max }^{+} \leq 1$ for $\bar{g}$, we deduce

$$
\left(\int_{M}|u|^{\frac{2 n}{n-2}} \mathrm{dvol}\right)^{\frac{n-2}{n}} \leq A \int_{M}\left(|\nabla u|^{2}+u^{2}\right) \mathrm{dvol}
$$

Applying Theorem 1.4 and Lemma 3.6 we then arrive at the desired inequality (1.22). 


\section{$4 W^{2, p}$ Sobolev Inequalities}

Proof of Theorem 1.8 Let $u \in W^{2, p}(M)$ for $1<p<\frac{\mu}{2}$. Since $(-\Delta+\Psi)^{\frac{1}{2}}$ is a pseudo-differential operator of order 1 [7] on a compact manifold, it is a bounded map from $W^{2, p}(M)$ into $W^{1, p}(M)$. Hence $v=(-\Delta+\Psi)^{\frac{1}{2}} u \in W^{1, p}(M)$. Applying Theorem 1.7 to $v$ we infer

$$
\|v\|_{\frac{\mu p}{\mu-p}} \leq C(\mu, A, p)\left\|(-\Delta+\Psi)^{\frac{1}{2}} v\right\|_{p}=C(\mu, A, p)\|(-\Delta+\Psi) u\|_{p},
$$

i.e.,

$$
\left\|(-\Delta+\Psi)^{\frac{1}{2}} u\right\|_{\frac{\mu p}{\mu-p}} \leq C(\mu, A, p)\|(-\Delta+\Psi) u\|_{p}
$$

For each $1<q<\infty,(-\Delta+\Psi)^{\frac{1}{2}}$ is a bounded operator from $W^{1, q}(M)$ into $L^{q}(M)$ with the bounded inverse $(-\Delta+\Psi)^{-\frac{1}{2}}$. Hence we deduce $u \in W^{1, \frac{\mu p}{\mu-p}}(M)$. Applying Theorem 1.7 to $u$ with the exponent $\frac{\mu p}{\mu-p}$ instead of $p$ we then infer

$$
\begin{aligned}
\|u\|_{\frac{\mu p}{\mu-2 p}} & \leq C\left(\mu, A, \frac{\mu p}{\mu-p}\right)\left\|(-\Delta+\Psi)^{\frac{1}{2}} u\right\|_{\frac{\mu p}{\mu-p}} \\
& \leq C\left(\mu, A, \frac{\mu p}{\mu-p}\right) C(\mu, A, p)\|(-\Delta+\Psi) u\|_{p} .
\end{aligned}
$$

(Note that $1<\frac{\mu p}{\mu-p}<\mu$ because $1<p<\frac{\mu}{2}$.)

Theorems 1.9 and 1.10 follow from Theorem 1.8, and [9, Theorem D] and [9, Theorem $\mathrm{D}^{*}$, respectively.

\section{Estimates of the Riesz Transform and $W^{1, p}$ Sobolev Inequalities}

The following theorem is a consequence of Bakry's result on $L^{p}$ estimates for the Riesz transform [1].

Theorem 5.1 Let $(M, g)$ be a complete Riemannian manifold (without boundary) of dimension $n \geq 2$ such that the Ricci curvature is bounded from below by $-a^{2}$ for some $0 \leq a<\infty$. Then there holds for each $1<p<\infty$

$$
\left\|(-\Delta+1)^{\frac{1}{2}} u\right\|_{p} \leq C(p)\left(\|\nabla u\|_{p}+(1+a)\|u\|_{p}\right)
$$

for all $u \in W^{1, p}(M)$, where the constant $C(p)$ depends only on $p$.

Proof In [1] the operator $-\Delta+\nabla \phi \cdot \nabla$ for a given function $\phi$ is handled. It is easy to see that all the arguments in [1] go through for the operator $-\Delta+1$. Hence [1, Theorem 4.1] extends to yield for $1<q<\infty$

$$
\|\nabla v\|_{q} \leq C_{q}\left(\left\|(-\Delta+1)^{\frac{1}{2}} v\right\|_{q}+a\|v\|_{q}\right)
$$


for all $u \in C_{c}^{\infty}(M)$, where $C_{q}$ depends only on $q$. On the other hand, we have $\left\|\mathrm{e}^{t(\Delta-1)} v\right\|_{q} \leq \mathrm{e}^{-t}\|v\|_{q}$ for $1<q<\infty$ and all $v \in L^{q}(M) \cap L^{2}(M)$. Applying the formula $(-\Delta+1)^{-\frac{1}{2}}=\Gamma\left(\frac{1}{2}\right)^{-1} \int_{0}^{\infty} t^{-\frac{1}{2}} \mathrm{e}^{t(\Delta-1)} \mathrm{d} t$ we infer $\left\|(-\Delta+1)^{-\frac{1}{2}} v\right\|_{q} \leq\|v\|_{q}$ and hence

$$
\|v\|_{q} \leq\left\|(-\Delta+1)^{\frac{1}{2}} v\right\|_{q}
$$

for all $v \in L^{q}(M) \cap L^{2}(M)$.

Since $(-\Delta+1)^{\frac{1}{2}}\left(C_{c}^{\infty}(M)\right)$ is dense in $L^{q}(M)$ (see [7,8]), we have for $u \in C_{c}^{\infty}(M)$, $1<p<\infty$ and $q=\frac{p}{p-1}$

$$
\begin{array}{r}
\left\|(-\Delta+1)^{\frac{1}{2}} u\right\|_{p}=\sup \left\{\left\langle(-\Delta+1)^{\frac{1}{2}} u,(-\Delta+1)^{\frac{1}{2}} v\right\rangle_{2}:\right. \\
\left.v \in C_{c}^{\infty}(M),\left\|(-\Delta+1)^{\frac{1}{2}} v\right\|_{q} \leq 1\right\} .
\end{array}
$$

But

$$
\begin{aligned}
\left\langle(-\Delta+1)^{\frac{1}{2}} u,(-\Delta+1)^{\frac{1}{2}} v\right\rangle_{2} & =\langle(-\Delta+1) u, v\rangle_{2}=\int_{M} \nabla u \cdot \nabla v+\int_{M} u v \\
& \leq\|\nabla u\|_{p}\|\nabla v\|_{q}+\|u\|_{p}\|v\|_{q} \\
& \leq\left(\|\nabla u\|_{p}+\|u\|_{p}\right)\left(\|\nabla v\|_{q}+\|v\|_{q}\right) .
\end{aligned}
$$

By (5.2) and (5.3) we then deduce $\|\nabla v\|_{q}+\|v\|_{q} \leq(1+(a+1) C(q))\left\|(-\Delta+1)^{\frac{1}{2}} v\right\|_{q}$. By (5.4) and (5.5) we then arrive at

$$
\left\|(-\Delta+1)^{\frac{1}{2}} u\right\|_{p} \leq C(p, a)\left(\|\nabla u\|_{p}+\|u\|_{p}\right),
$$

where $C(p, a)=1+(a+1) C_{\frac{p}{p-1}}$. By (5.2), (5.3) (applied to $p$ ) and (5.6) we conclude that $(M, g)$ is $(1, p)$-Bessel and that (5.6) holds true for all $u \in W^{1, p}(M)$.

To derive the inequality (5.13), we consider the metric $\bar{g}=\lambda^{2} g$, where $\lambda=1+a$. Since the Ricci curvature of $\bar{g}$ is bounded from below by $-\frac{a^{2}}{(1+a)^{2}} \geq-1$, we have by (5.6)

$$
\left\|\left(-\Delta_{\bar{g}}+1\right)^{\frac{1}{2}} u\right\|_{p, \bar{g}} \leq C(p, 1)\left(\left\|\nabla_{\bar{g}} u\right\|_{p, \bar{g}}+\|u\|_{p, \bar{g}}\right)
$$

for $1<p<\infty$ and all $u \in L^{p}(M)$. But $\Delta_{\bar{g}}=\lambda^{-2} \Delta$. Hence we obtain

$$
\left\|\left(-\Delta+\lambda^{2}\right)^{\frac{1}{2}} u\right\|_{p, \bar{g}} \leq \lambda C(p, 1)\left(\left\|\nabla_{\bar{g}} u\right\|_{p, \bar{g}}+\|u\|_{p, \bar{g}}\right)
$$

Transforming to $g$ we obtain

$$
\left\|\left(-\Delta+\lambda^{2}\right)^{\frac{1}{2}} u\right\|_{p} \leq C(p, 1)\left(\|\nabla u\|_{p}+\lambda\|u\|_{p}\right) .
$$


By (3.33) there holds

$$
\begin{aligned}
\left\|\left(-\Delta+\lambda^{2}\right)^{\frac{1}{2}} u\right\|_{p} & \geq c_{1}\left(\lambda\|u\|_{p}+\left\|(-\Delta)^{\frac{1}{2}} u\right\|_{p}\right) \geq c_{1}\left(\|u\|_{p}+\left\|(-\Delta)^{\frac{1}{2}} u\right\|_{p}\right) \\
& \geq c_{1} c_{2}^{-1}\left\|(-\Delta+1)^{\frac{1}{2}} u\right\|_{p} .
\end{aligned}
$$

It follows that

$$
\left\|(-\Delta+1)^{\frac{1}{2}} u\right\|_{p} \leq c_{1}^{-1} c_{2} C(p, 1)\left(\|\nabla u\|_{p}+\lambda\|u\|_{p}\right)
$$

which gives rise to $(5.13)$.

The next result is a consequence of the $L^{p}$ estimates for the Riesz transform due to Li [5].

Theorem 5.2 Let $(M, g)$ be a complete Riemannian manifold (without boundary) of dimension $n \geq 2$. Assume that there is a constant $c_{1}$ such that

$$
\left\|e^{t(\Delta-1)} u\right\|_{\infty} \leq c_{1} t^{-\frac{n}{2}}\|u\|_{1}
$$

for all $u \in L^{1}(M)$ and $0<t \leq 1$. Assume that $\left(\operatorname{Ric}_{\min }+c_{2}\right)^{-} \in L^{\frac{n}{2}+\epsilon}(M)$ for some $c_{2} \geq 0$ and $\epsilon>0$. Then there holds for each $1<p<2$

$$
\left\|(-\Delta+1)^{\frac{1}{2}} u\right\|_{p} \leq C\left(\|\nabla u\|_{p}+(1+\gamma)\|u\|_{p}\right)
$$

for all $u \in W^{1, p}(M)$, where

$$
\gamma=\left(\int_{M}\left|\left(\mathrm{Ric}_{\min }+c_{2}\right)^{-}\right|^{\frac{n}{2}+\epsilon} \mathrm{dvol}\right)^{\frac{1}{2 \epsilon}}
$$

and the constant $C$ can be bounded above in terms of upper bounds for $n, c_{1}, c_{2}, \frac{1}{\epsilon}$ and $\frac{1}{p-1}$.

Proof This follows from the proof of Theorem 2.2 in [5] and the arguments in the above proof of Theorem 5.1.

Proof of Theorems 1.11-1.13 Combine Theorem 5.1 with Theorems 1.4-1.6.

Proof of Theorems 1.14-1.16 Combine Theorem 5.2 with Theorems 1.4-1.6, and also apply Theorem 3.1 and the arguments in the proof of Theorem 1.7.

\section{References}

1. Bakry, D.: Étude des transfromations de Riesz dans les variété riemanniennes à courbure de Ricci minorée, Séminaire de Probabilités, vol. XXI, pp. 137-172. Lecture notes in mathematics, vol. 1247. Springer, Berlin (1987) 
2. Gallot, S.: Inégalités isopérimétriques et analytiques sur les variétés riemanniennes. Astérisque 163164, 31-91 (1988)

3. Gallot, S.: Isoperimetric inequalities based on integral norms of Ricci curvature. Astérisque 157-158, 191-216 (1988)

4. Gilbarg, D., Trudinger, N.S.: Elliptic Partial Differential Equations of Second Order, 2nd edn. Springer, New York (1983)

5. Li, X.D.: Riesz transforms for symmetric diffusion operators on complete Riemannian manifolds. Rev. Mat. Iberoamericana 22(2), 591-648 (2006)

6. Perelman, G.: Ricci flow with surgery on three-manifolds. arXiv.math/0303109v1 (2003). Accessed March 2003

7. Seeley, R.T.: Complex powers of an elliptic operator. Proc. Symp. Pure Math. 10, 288-307 (1967)

8. Strichartz, R.: Analysis of the Laplacian on the complete Riemannian manifold. J. Funct. Anal. 52, 48-79 (1983)

9. Ye, R.: The logarithmic Sobolev inequality along the Ricci flow. arXiv:math/0707.2424

10. Ye, R.: The logarithmic Sobolev inequality along the Ricci flow in dimension 2. arXiv:0708.2003

11. Ye, R.: The logarithmic Sobolev inequality along the Ricci flow: the case $\lambda_{0}\left(g_{0}\right)=0$. arXiv:0708.2005

12. Ye, R.: The log entropy functional along the Ricci flow. arXiv:0708.2008 